\title{
Aprendizaje basado en proyectos aplicados en la asignatura de materiales de construcción
}

\author{
Camila A. Burgos-Leiva1, José A. Rementeria-Piñones², Jaime C. Espinoza-Oyarzún' y Arturo B. Rodríguez-García1 \\ (1) Universidad de Santiago de Chile, Depto. de Tecnologías Industriales, Grupo de Investigación en Nuevas \\ Tecnologías (GINT), Santiago, Chile. (correo-e: camila.burgos.l@usach.cl; arturo.rodriguez@usach.cl; \\ jaime.espinoza@usach.cl). \\ (2) Universidad de Santiago de Chile, Depto. de Publicidad e Imagen, Santiago, Chile. \\ (correo-e: jose.rementeria@usach.cl).
}

Recibido Jul. 17, 2020; Aceptado Sep. 14, 2020; Versión final Nov. 25, 2020, Publicado Abr. 2021

\begin{abstract}
Resumen
El objetivo de este estudio es implementar la metodología de aprendizaje basado en proyectos (ABPy) con la finalidad de mejorar la apropiación de conocimientos, las habilidades de trabajo en equipo y la colaboración entre estudiantes. La metodología se implementa en la asignatura de materiales de construcción de la carrera tecnología en construcciones de la Universidad de Santiago de Chile. Para este propósito, se realizó el secado de madera de pino radiata y pino oregón para observar las diferencias de masas en un lapso de 48 horas. En cuanto al aprendizaje estudiantil en base al análisis de las calificaciones finales obtenidas, el aumento de aprendizaje grupal alcanza al 39,1\%, tomando en cuenta el promedio de entrada de los estudiantes. La valoración por el entorno y la responsabilidad de la vida comunitaria se manifestó en las intervenciones en el aula. Se concluye que las habilidades de trabajo en equipo y colaborativo de los estudiantes mejoraron significativamente.
\end{abstract}

Palabras clave: educación tecnológica; aprendizaje basado en proyectos; educación ambiental; materiales

\section{Applied project-based learning in a construction materials course}

\begin{abstract}
The primary objective of this research study was to implement a project-based learning (PBL) methodology to improve knowledge appropriation, teamwork skills, and collaboration between students. The methodology was applied by using an in-class experiment in the building materials course of the technology in construction degree at the University of Santiago of Chile. For the in-class experiment, Radiata and Oregon pinewoods were dried for 48 hours to assess changes in mass. The results showed that in-group learning increased $39.1 \%$ after taking into account student final grades and average entry grades. Student environmental assessment and student community life commitments were shared in classroom interventions. It is concluded that student teamwork and collaborative skills improved significantly.
\end{abstract}

Keywords: technological education; project based learning; environmental education, materials 


\section{INTRODUCCIÓN}

El aprendizaje basado en proyectos (ABPy), es una forma de instrucción centrada en el estudiante que tiene principios basados en el constructivismo: el aprendizaje es específico del contexto, las y los alumnos participan activamente en el proceso de aprendizaje y logran sus objetivos a través de interacciones sociales y el intercambio de conocimientos y comprensión (Kokotsaki et al, 2016). Se puede considerar un tipo particular de aprendizaje basado en la investigación donde el contexto del aprendizaje se proporciona a través de preguntas y problemas auténticos dentro de las prácticas del mundo real que conducen a experiencias de aprendizajes significativos (Wurdinger et al, 2007). En la educación superior chilena el ABPy se ha utilizado en la formación de diferentes ingenierías (Villalobos-Abarca et al, 2018; Herrera, 2017), también en el área de la enseñanza de la medicina veterinaria y en biología (Mateos et al, 2020), en cursos de geología se incorporó el ABPy y la gamificación como metodologías de enseñanza-aprendizaje innovadoras (RodríguezOroz et al, 2019).

El competitivo mercado laboral está demandando a profesionales que no sólo tengan competencias de índole técnica, sino que además tengan la capacidad de adaptarse a los cambios, que sean permeables, persuasivos y flexibles, capaces de liderar personas y por tanto gestionar emociones. La Universidad de Santiago de Chile, a través de su Modelo Educativo Institucional, es una institución generadora de factores de socialización que incorpora dentro del currículum formativo de cada especialidad un sello institucional que promueve a los docentes a formar a las y los estudiantes en el desarrollo de sus habilidades socio-emocionales. El propósito es formar titulados universitarios, capaces de enfrentarse a los retos del mercado laboral de una forma cognitiva y emocionalmente saludable.

La asignatura Materiales de Construcción, fue seleccionada debido a su importancia dentro del currículum de la carrera de Tecnología en Construcciones de la Universidad de Santiago de Chile, además que posee las características teórico - práctica (posee 4 horas de práctica y 2 horas de teoría), en donde los y las estudiantes pueden desarrollar variadas habilidades que serán de gran utilidad en su vida profesional. Las exigencias del mundo actual vienen marcadas por la creciente búsqueda del desarrollo del potencial humano en los diferentes ámbitos sociales y profesionales. En dicha asignatura, se realizan prácticas de laboratorio sobre variadas materias y materiales, entre ellas es relevante el tema de la madera; específicamente la práctica relacionada con aquellas características tales como; humedad, volumen y densidad de este material. Los estudios indican que las prácticas de laboratorio pueden ser utilizadas como una estrategia didáctica que desde el paradigma constructivista promueve la construcción de conocimiento científico en los estudiantes (Windschitl, 2002).

Es de relevancia que el Tecnólogo en Construcciones adquiera e internalice los conceptos de contenido de humedad y densidad de madera en general, debido a que es un material el cual se utiliza ampliamente en la construcción y, al poseer propiedades higroscópicas, tiene la capacidad de absorber y liberar agua con facilidad, así también como posee una variación dimensional debido a esta característica. Es importante recalcar que en la actualidad hay una gran cantidad de contaminación ambiental provocada por el uso de leña húmeda para calefacción del hogar, la cual podría ser mitigada por un buen uso de madera (leña), ya sea la que proviene de aserraderos certificados como de productores locales de leña, los cuales podrían tener herramientas, de fácil uso, para verificar la humedad de la madera y que esta genere niveles menores de contaminación ambiental. En la actualidad, se ha verificado que la combustión de la leña es la mayor fuente de material particulado fino en las grandes ciudades del centro y sur de Chile (González et al, 2019).

Actualmente, el proceso educativo que se lleva a cabo en los laboratorios de la carrera de Tecnología en Construcciones es altamente competitivo e individual, lo que hace necesario desarrollar en el estudiante habilidades colaborativas y cooperativas. Los y las estudiantes deben ser convocados en temas que son de importancia cotidiana para que tenga sentido útil y que les sugiera pertinencia, en directa relación con la responsabilidad social universitaria.

En este contexto la contaminación ambiental cumple con este requisito, ya que es un tema que afecta a todos los chilenos de forma transversal, no tan solo en la Región Metropolitana de Santiago, sino que también en el resto del país. Uno de los elementos contaminantes es el mal uso de la leña como material de calefacción, provocando una contaminación la cual está directamente relacionada con el contenido de humedad que esta posee, debido a que a mayor humedad mayor es la cantidad de gases tóxicos que puede generar al hacer la combustión (Fine et al, 2002). La utilización de la leña como calefacción se debe “... a su precio, factibilidad de acceso y costumbre, la leña es el principal combustible utilizado en las ciudades del sur del país. Esto ha incidido en altos niveles de contaminación, los cuales ponen en riesgo la vida de la población y tienen efectos directos tanto en mortalidad como morbilidad." (Rivera y Lima, 2018:26). Esto explica porque es el principal combustible en la mayoría de los hogares, especialmente en la zona centro sur y sur de Chile (Reyes et al, 2019). 
La ciudad de Temuco tiene índices elevados de contaminación ambiental debido a la gran utilización de leña como material de calefacción (Cereceda-Balic et al, 2012). Además, cerca del 93\% del material particulado originado a partir de la combustión de leña corresponde a material particulado cuyo diámetro es menor a 2,5 micrones (MP2,5), partículas que presentan efectos especialmente nocivos sobre la salud humana (Cortés y Ridley, 2013). El problema del mal uso de la leña es real y significativo, actualmente es el responsable de la mayor cantidad de material particulado en el medio ambiente urbano.

Los factores principales que generan episodios críticos de contaminación atmosférica urbana son: a) Viviendas: la mala aislación térmica de las viviendas genera que el gasto de energía en combustión sea más elevado, aumentando de forma innecesaria las emisiones de material particulado. (Cortés y Ridley, 2013); b) Calefactores: Las estufas en mal estado, muchas veces artesanales, provocan que la emisión de material particulado no regulado, actualmente se debe generar un máximo de 2.5 gramos, principalmente la contaminación aumenta cuando la estufa está utilizando el tiraje cerrado, práctica habitual en Chile. (Schueftan et al, 2016); y c) Inversión térmica: La contribución a la contaminación se genera cuando se utilizan las estufas con el tiraje cerrado lo cual permite que se acumulen una gran cantidad de contaminantes en la atmósfera superficial de la ciudad, amplificando el impacto negativo de la ineficiencia de nuestros calefactores y viviendas. (Schueftan et al, 2016).

Cuando en las décadas del 80 y 90 comenzaron los episodios de contaminación del aire en Santiago nadie propuso prohibir la gasolina, por el contrario, se propuso mejorar la eficiencia de los vehículos, la calidad del combustible y restringir la circulación de los no catalíticos, entre otras medidas (Romero et al, 2010). Lo mismo se debe hacer con la leña, mejorar los calefactores, hacer más eficientes las viviendas y mejorar el combustible; en el caso de la leña, debe cumplir con estándares de calidad como contenido de humedad, densidad, dimensionamiento y especie.

La metodología y temática, llevada a cabo en el laboratorio, activa en los estudiantes potencialidades que se espera se conviertan en habilidades, que tendrán como consecuencia inmediata, el tratamiento de temas de forma lúdica y operativa, la cooperación al interior del grupo y la colaboración grupal. Este método facilita la entrega de contenido y genera aprendizaje significativo con responsabilidad social. Con las habilidades que se desarrollan utilizando la metodología del ABPy genera un efecto sustancial en las capacidades de los alumnos tanto para su formación educacional como su formación profesional, obteniendo habilidades de trabajo en equipo, colaboración y cooperación. Las calificaciones de las tres últimas versiones de la asignatura Materiales de Construcción, muestran un buen porcentaje de aprobación, tal como se puede apreciar en la Tabla 1. Sin embargo, no existe evidencia de mejora del trabajo en equipo, como tampoco en habilidades colaborativas y cooperativas.

Tabla 1: Distribución del porcentaje de aprobación de estudiantes de la asignatura Materiales de Construcción

\begin{tabular}{|c|c|c|}
\hline Año/semestre & $\%$ & $\begin{array}{c}N \text { (cantidad de } \\
\text { estudiantes) }\end{array}$ \\
\hline $2016-1$ & 76,47 & 18 \\
\hline $2016-2$ & 84,20 & 19 \\
\hline $2017-1$ & 84,60 & 13 \\
\hline $2017-2$ & 80,40 & 41 \\
\hline $2018-1$ & 77,70 & 18 \\
\hline $2018-2$ & 72,90 & 37 \\
\hline
\end{tabular}

El presente artículo busca promover el trabajo en equipo y el desarrollo de habilidades de cooperación y colaboración en estudiantes de la Universidad de Santiago de Chile (USACH). De las metodologías activas existentes en la literatura la que mejor desarrolla estas habilidades es el ABPy o Aprendizaje Basado en Proyectos (Zancul et al, 2017), por estas razones el equipo docente optó por tal metodología, esperando establecer dicha intervención de forma permanente en la asignatura. El proyecto incorpora la difusión de resultados que servirá de base para futuras réplicas en otras asignaturas de materiales de construcción en la carrera de Tecnología en Construcciones. 


\section{METODOLOGÍA}

El problema expuesto a los estudiantes se basó en la medición de humedad en las piezas de madera antes y después de ser sometida a la acción del calor en la estufa. La comprobación de contenido de humedad que posee la leña antes de ser utilizada en la estufa, se realizó con instrumentos tradicionales de medición de humedad y de peso de la muestra; esta medición se hizo antes y durante 48 horas de secado en horno a temperatura constante según normativa de ensayo $\left( \pm 103^{\circ} \mathrm{C}\right)$, (ASTM, 2020); de esta forma, se pudo comprobar el porcentaje de humedad inicial que contenía la madera. La experiencia se realizó con 3 grupos (G1, G2, G3) de estudiantes en tiempos diferenciados, tal como se puede observar en la Figura 1. La primera etapa fue el análisis de los indicadores de medición de cooperación, colaboración y trabajo en equipo, para poner en práctica los 9 pasos que contempla el ABPy que se especifica más adelante. Para finalmente realizar la post medición $(\mathrm{C}=$ Calificación, $\mathrm{A}=\mathrm{Actitud})$ y análisis de los resultados en comparación con los primeros indicadores.

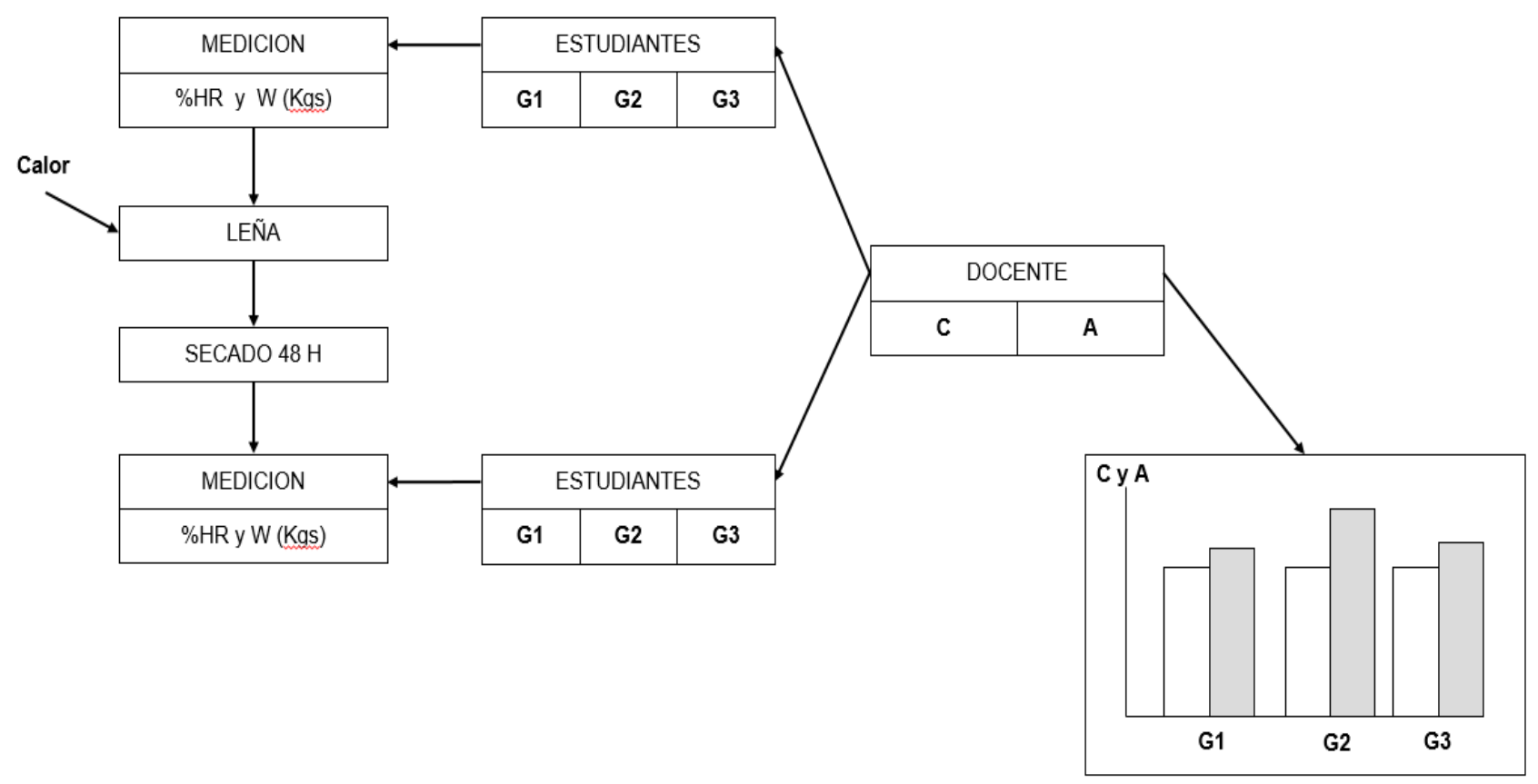

Fig. 1: Esquematización metodológica.

De acuerdo con Badia y García (2006) en el diseño, planificación e implementación de un proyecto colaborativo se pueden identificar una serie de etapas que el equipo docente debe tener en cuenta desde el principio, a saber, son: 1) selección del tema y planteamiento de pregunta guía, donde se genera la motivación por la realización de la experiencia. Además de entregar las herramientas para que desarrollen trabajo autónomo, 2) formación de grupos de trabajo afines, 3) planificación del trabajo, establecer los objetivos, el calendario y distribución de las tareas, 4) investigación hacer lo requerido para alcanzar los objetivos compartidos, 5) análisis y síntesis aplicando estrategias enfocadas a la consecución de los objetivos, 6) elaboración del producto en base a las orientaciones y apoyo del equipo docente, 7) presentación del producto en base a los resultados del proyecto, 8) respuesta colectiva a la pregunta inicial: entrega de informe escrito, 9) evaluación: presentación y defensa ante el equipo docente y autoevaluación del grupo.

Se eligió la asignatura de Materiales de Construcción del periodo académico 2019-1, que contaba con 17 estudiantes. Para llevar a efecto las mediciones antes mencionadas, se dividió a los estudiantes en tres grupos de manera aleatoria; la experiencia fue realizada de manera seriada, es decir, un grupo a la vez, pero sin que los grupos no ejecutantes estuvieran presentes. Las instrucciones fueron entregadas en una guía escrita, en donde además se incorporó pauta y rúbrica para cada estudiante; durante la experiencia solo se encontraban presentes los dos docentes a cargo de la experiencia, que utilizaron 10 minutos para introducir el tema, luego se mantuvieron en el rol de observadores.

La experiencia de laboratorio fue conformada por 3 grupos elegidos de forma aleatoria por los docentes según listado del curso, los cuales quedaron conformados por 2 grupos con 6 alumnos/as y 1 con 5 alumnos/as, en cada grupo todos y todas las y los estudiantes debían tener un rol específico; encargados de las mediciones, de los registros, del funcionamiento de los equipos, redacción y tabulación de los resultados, cada uno de estos roles deben interactuar entre sí y a su vez retroalimentarse de la información que entrega cada uno. Se 
realizó una evaluación a la entrada y salida de la experiencia, basado en una evaluación compuesta por dos parámetros, el primero es el nivel de conocimiento y el segundo la actitud y la participación al interior de su grupo frente a la actividad, utilizando una escala de notas 1 a 7.

La variable calificación hace referencia a la evaluación que se practica de manera habitual en la asignatura y pretende establecer el nivel de conocimiento adquirido por los estudiantes, mientras que la variable actitud y participación es una evaluación observacional previamente consensuada con el equipo docente, cabe mencionar que la evaluación de actitud y participación no es realizada por el docente que impartió la clase para evitar sesgo en la observación.

\section{Herramientas Virtuales}

Las observaciones se realizaron indirectamente e individualmente, mediante un website propietario perteneciente a la Universidad, su acceso es: http://academica2.usach.cl/ basado en Moodle. Todos los profesores y estudiantes se encuentran registrados en la plataforma. Cuenta con un desarrollo de herramientas para interactuar entre estudiantes y profesores de forma síncrona y asíncrona. Toda la información de la asignatura está disponible con antelación para los estudiantes, y cuentan con elementos de evaluación online de manera asíncrona. Todos los estudiantes y profesores cuentan con acceso a internet tanto en sus hogares como en la Universidad, donde pueden disponer de tiempo de acceso indefinido.

\section{RESULTADOS Y ANÁLISIS}

En la Figura 2, se puede observar la caracterización de los estudiantes, en el primer caso no parece existir mucha diferencia en la muestra está distribuida con una relativa equidad. Sin embargo, existe en el ingreso dos semestres predominantes, tales como el 2019-1 y 2018-1. El total de estudiantes asciende a 17, y todos pertenecen a comunas del sur de la Región Metropolitana de Chile. Se define la variable: Traspaso, como aquellos estudiantes que vienen de otra carrera, facultad y/o universidad. La variable: Prueba de Selección Universitaria (PSU), aquellos estudiantes que ingresan a la carrera con el test estandarizado escrito, implementado en Chile desde 2003, para el proceso de admisión a la educación universitaria.

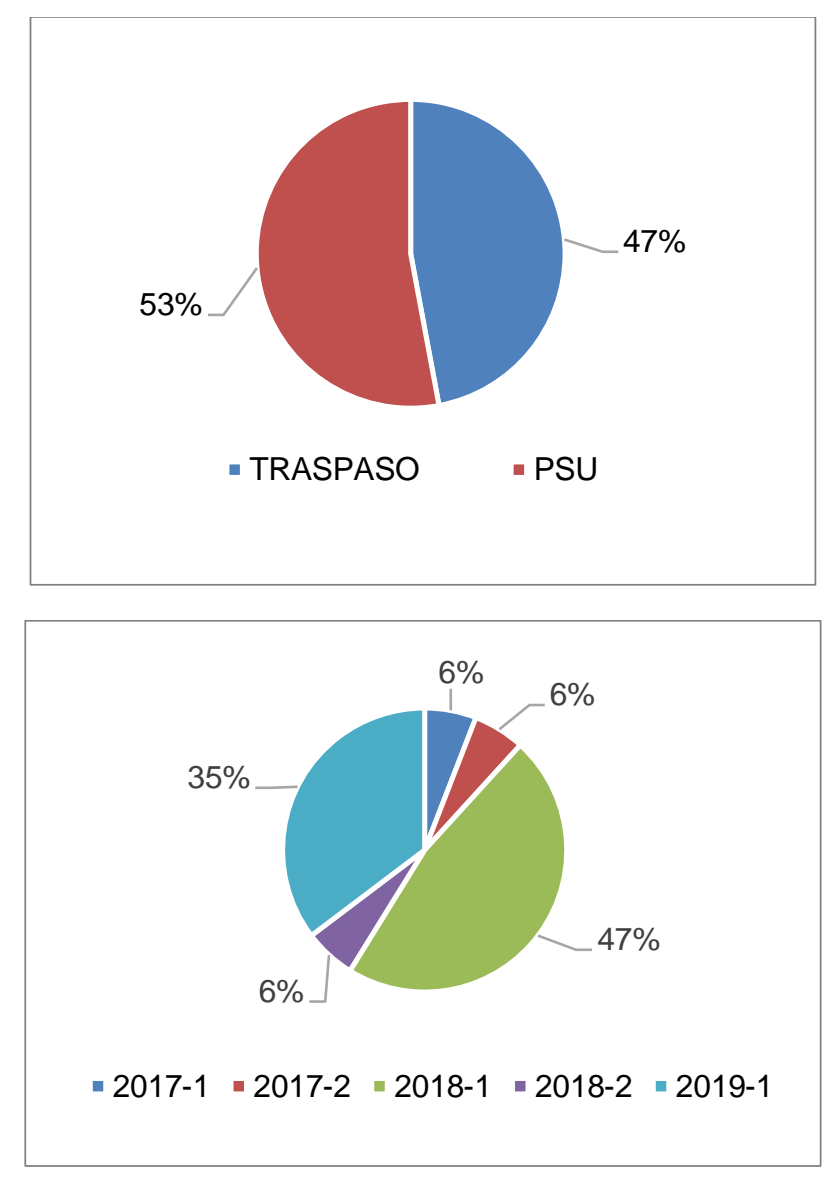

Fig. 2: Caracterización de la muestra estudiantil. 
La Tabla 2, muestra los resultados ordenados por grupo y con las mediciones individuales al inicio y final de la intervención. En la Tabla 3, podemos observar la comparación del incremento promedio de los grupos, hubo una mejora significativa en todos los grupos. El grupo 3 obtuvo un alza mayor en su desempeño, luego el grupo 2 y al final el grupo 1. En las mediciones de comparación entre la Calificación y la Actitud del grupo 2, fue similar en ambos incrementos. El grupo 1 logró subir de relativa significancia en la Actitud por sobre la Calificación y el grupo 3 logró un incremento muy significativo en Actitud frente a la Calificación.

En la Tabla 3, podemos observar el aumento del promedio grupal de Calificaciones y la valoración de la Actitud, es visible la mejora en todos los grupos después de la experiencia, la metodología activa logró aumentar el desempeño de los estudiantes que es concordante con los resultados obtenidos en experiencias realizadas en Brasil, que se derivaron de la estructura innovadora del programa, así como de su adopción de metodologías de aprendizaje activo como ABPy, que impulsan el desarrollo de los estudiantes hacia la excelencia. (Monteiro et al, 2017).

Tabla 2: Evaluaciones Individuales de Calificación y Actitud

\begin{tabular}{|c|c|c|c|c|c|c|}
\hline \multirow{2}{*}{ Grupo } & \multirow{2}{*}{$N$} & \multicolumn{2}{|c|}{ Inicio } & \multicolumn{2}{|c|}{ Final } & \multirow{2}{*}{$\frac{\text { Diferencia }}{\text { Promedio }}$} \\
\hline & & Calificación & Actitud & Calificación & Actitud & \\
\hline \multirow{6}{*}{ G1 } & 1 & 4.8 & 3 & 4 & 5 & 15.38 \\
\hline & 2 & 3.5 & 4 & 4.8 & 6 & 44 \\
\hline & 3 & 4.8 & 2 & 7 & 4.5 & 69.12 \\
\hline & 4 & 3.5 & 4 & 4.8 & 4 & 17.33 \\
\hline & 5 & 5.5 & 2 & 6.3 & 5 & 50.67 \\
\hline & 6 & 2.5 & 4 & 6.3 & 5 & 73.85 \\
\hline \multirow{6}{*}{ G2 } & 7 & 4 & 4 & 6.3 & 5 & 41.25 \\
\hline & 8 & 4 & 3 & 6.3 & 5 & 61.43 \\
\hline & 9 & 4 & 4 & 6.3 & 5 & 41.25 \\
\hline & 10 & 4.8 & 2 & 6.3 & 5.3 & 70.59 \\
\hline & 11 & 3 & 4 & 4 & 5.5 & 35.71 \\
\hline & 12 & 3.5 & 4 & 4.8 & 6 & 44 \\
\hline \multirow{5}{*}{ G3 } & 13 & 4.8 & 4 & 6.3 & 6 & 39.77 \\
\hline & 14 & 4 & 3 & 6.3 & 5 & 61.43 \\
\hline & 15 & 4.8 & 2 & 6.3 & 5 & 66.18 \\
\hline & 16 & 3.5 & 3 & 3 & 6 & 38.46 \\
\hline & 17 & 2.5 & 4 & 4.8 & 6.5 & 73.85 \\
\hline
\end{tabular}

Tabla 3: Comparación de Promedio Grupal de Calificación y Actitud de Inicio y Final de la Intervención

\begin{tabular}{|c|c|c|c|c|c|c|c|c|}
\hline \multirow{2}{*}{ Grupos } & \multicolumn{4}{|c|}{ Promedio Calificación } & \multicolumn{4}{c|}{ Promedio Actitud } \\
\cline { 2 - 9 } & Inicio & Final & $\begin{array}{c}\text { Diferencia en } \\
\text { puntos }\end{array}$ & $\begin{array}{c}\text { Diferencia } \\
\text { porcentual }\end{array}$ & Inicio & Final & $\begin{array}{c}\text { Diferencia en } \\
\text { puntos }\end{array}$ & $\begin{array}{c}\text { Diferencia } \\
\text { porcentual }\end{array}$ \\
\hline G1 & 4,10 & 5,53 & 1,43 & 20,48 & 3,17 & 4,92 & 1,75 & 25,00 \\
\hline G2 & 3,88 & 5,67 & 1,78 & 25,48 & 3,50 & 5,30 & 1,80 & 25,71 \\
\hline G3 & 3,92 & 5,34 & 1,42 & 20,29 & 3,20 & 5,70 & 2,50 & 35,71 \\
\hline
\end{tabular}


También, en la Tabla 3, es visible el aumento del rendimiento tanto en Calificación como en Actitud. En la Tabla 2, por otro lado, podemos notar que en los tres grupos hay estudiantes que inicialmente tenían mejor Actitud que Calificación, sin embargo, al final de la experiencia los resultados fueron mejores, esto lleva a pensar que aquellos estudiantes que tienen; alta en Actitud, tienden a aprovechar mejor la metodología (Ferreira \& Trudel, 2012). Por otro lado, se observa en los tres grupos la diferencia positiva de las calificaciones antes y después de la experiencia. Al realizar la comparación individual al interior de los grupos, que se puede observar en la Tabla 2, de las calificaciones y la valoración de la actitud que la mayoría logró un incremento después de la experiencia. La comparación de las calificaciones y la valoración de la actitud tanto del grupo 2 como del grupo 3 , se puede observar la mejora después de la experiencia.

En la Tabla 4, se muestra las seis pruebas de independencia que se realizaron para INGRESO que corresponden a los semestres de ingreso al programa constituido por los semestres y TIPO formada por los estudiantes de traspaso o ingreso PSU. Se observa la clara dependencia de los resultados al ingreso tanto al inicio como al final, lo que determina que las habilidades y conocimiento pudieron ser previos, mientras que no existe una clara dependencia por el tipo de ingreso.

Tabla 4: Pruebas de independencia $\left(\chi^{2}, 95 \%\right)$

\begin{tabular}{|c|c|c|c|c|}
\hline & \multicolumn{2}{|c|}{ Inicio } & \multicolumn{2}{c|}{ Final } \\
\hline & Calificación & Actitud & Calificación & Actitud \\
\hline Ingreso & Dependiente & Dependiente & Dependiente & Independiente \\
\hline Tipo & Independiente & Independiente & Independiente & Independiente \\
\hline
\end{tabular}

\section{CONCLUSIONES}

Con la aplicación de esta metodología basada en proyectos y de forma muy activa con el estudiante, se mejoró las habilidades de cooperación e inducir las habilidades colaborativas, para poder ayudar a mejorar el trabajo en equipo, conforme al Modelo Educativo Institucional USACH (MEI); que fue realizado a través de las actividades que se han propuesto en el laboratorio en donde los estudiantes debieron realizar la experiencia de humedad de la madera (leña) en conjunto con sus compañeros como equipo de laboratorio. Para poder identificar la colaboración se utilizó la plataforma virtual Moodle, en donde se pudo evidenciar la actividad de cada uno de los estudiantes en el transcurso de la experiencia y como ellos aportaron al trabajo en equipo. Es importante indicar que el aumento de la actividad en las comunicaciones asíncronas es relevante para lograr colaboración entre los estudiantes.

El cambio realizado en la forma de ejecución de esta experiencia práctica en el laboratorio de Materiales de Construcción, implicó un cambio en la metodología permitiendo una participación muy activa de los y las estudiantes con una constante supervisión y retroalimentación del docente. La metodología orientada a proyectos es un motor de actividades apoyado por el uso constante del conocimiento adquirido, por lo que dicho conocimiento se ancla en los estudiantes, logrando mejorar los resultados respecto de la metodología convencional. Como valor agregado a estas metodologías se espera que los alumnos y las alumnas empaticen y tomen conciencia sobre el buen uso de la leña como material combustible para la calefacción.

\section{AGRADECIMIENTOS}

Especial agradecimiento a los investigadores del Proyecto de Innovación Docente Código 069-2017, al Laboratorio de Simulación en Madera del Departamento de Tecnologías Industriales y a la Facultad Tecnológica de la Universidad de Santiago de Chile, por el importante apoyo al desarrollo del estudio.

\section{REFERENCIAS}

ASTM, D4442-20, Standard Test Methods for Direct Moisture Content Measurement of Wood and Wood-Based Materials. https://dx.doi.org/10.1520/D4442-20, ASTM International: West Conshohocken, Pensilvania (2020)

Badia, A. y García, C. Incorporación de las TIC en la Enseñanza y el Aprendizaje Basados en la Elaboración Colaborativa de Proyectos. http://dx.doi.org/10.7238/rusc.v3i2.286, RUSC. Universities and Knowledge Society Journal, 3(2), 42-54 (2006)

Cereceda-Balic, F., Fadic, X., y otros tres autores, Obtaining Polycyclic Aromatic Hydrocarbon Concentration Ratios and Molecular Markers for Residential Wood Combustion: Temuco, a Case Study. https://doi.org/10.1080/10473289.2011.617599, Journal of the Air \& Waste Management Association, 62(1), 44-51, (2012) 
Cortés, A., y Ridley, I., Efectos de la Combustión a Leña en la Calidad del Aire Intradomiciliario: La Ciudad de Temuco como Caso de Estudio. http://dx.doi.org/10.4067/S0718-83582013000200008, Revista INVI, 28(78), 257-271 (2013)

Ferreira, M., y Trudel, A., The Impact of Problem-Based Learning (PBL) on Student Attitudes Toward Science, ProblemSolving Skills, and Sense of Community in the Classroom, The Journal of Classroom Interaction, 47(1), 23-30 (2012)

Fine, P.M., Cass, G.R. y Simoneit, B.R.T., Chemical Characterization of Fine Particle Emissions from the Fireplace Combustion of Woods Grown in the Southern United States. https://doi.org/10.1021/es0108988, Environmental Science and Technology, 36(7), 1442-1451 (2002)

González, P., Zavieso, L., y otros cinco autores, Evaluación Programa de Recambio de Calefactores a Leña del Ministerio del Medio Ambiente. Informe Final. http://www.dipres.gob.cl/597/articles-187242_informe_final.pdf, Santiago, Chile: Universidad de Chile, Centro de Sistemas Públicos (2019)

Herrera, R. F., Collaborative Project-Based Learning of Environments Programming From Civil Engineering Projects. https://doi.org/10.15359/ree.21-2.10, Revista Electrónica Educare, 21(2),205-222 (2017)

Kokotsaki, D., Menzies, V., Wiggins, A., Project-based Learning: a Review of the Literature. https://doi.org/10.1177/1365480216659733, Improving schools, 19 (3), 267-277 (2016)

Mateos, E., Redondo, S., y otros tres autores, Implantación de una Metodología Docente Activa en la Asignatura de Redacción y Ejecución de Proyectos del Grado en Biología. http://dx.doi.org/10.21703/rexe.20201939mateos15, Revista de estudios y experiencias en educación, 19(39), 259-274 (2020)

Monteiro, S. B. S., Reis, A. C. B., y otros dos autores, A Project-based Learning Curricular Approach in a Production Engineering Program. https://doi.org/10.1590/0103-6513.226116, Production, 27(spe), e20162261 (2017)

Reyes, R., Schueftan, A y otros dos autores, Controlling Air Pollution in Context of High Energy Poverty Levels in Southern Chile: Clean Air But Coulder Houses?. https://doi.org/10.1016/j.enpol.2018.10.022, Energy Policy, 124, pp. 301-311 (2019)

Rivera, J y., Lima, J., Análisis del Costo de Sustituir Consumo de Leña por Gas Natural, Gas Licuado de Petróleo, Kerosene o Pellets. Informe Final. https://docplayer.es/108374731-Analisis-del-costo-de-licuado-de-petroleo-kerosene-opellets.html, Santiago, Chile, Universidad de Chile, Intelis (2018)

Rodríguez-Oroz, D., Gómez-Espina, R., y otros dos autores, Aprendizaje Basado en un Proyecto de Gamificación: Vinculando la Educación Universitaria con la Divulgación de la Geomorfología de Chile. https://revistas.uca.es/index.php/eureka/article/view/4418/5371, Revista Eureka sobre Enseñanza y Divulgación de las Ciencias, 16 (2), 2202-2215 (2019)

Romero, H., Irarrázaval, F., y otros tres autores, Climas Urbanos y Contaminación Atmosférica en Santiago de Chile. http://dx.doi.org/10.4067/S0250-71612010000300002, EURE (Santiago), 36(109), 35-62 (2010)

Schueftan, A.; Sommerhoff, J. \& González, A. D., Firewood Demand and Energy Policy in South-Central Chile. https://doi.org/10.1016/j.esd.2016.04.004, Energy for Sustainable Development, 33, 26-35 (2016)

Villalobos-Abarca, M., Herrera-Acuña, R., y otros dos autores, Aprendizaje Basado en Proyectos Reales Aplicado a la Formación del Ingeniero de Software. http://dx.doi.org/10.4067/S0718-50062018000300097, Formación universitaria, 11(3), 97-112 (2018)

Windschitl, M., Framing Constructivism in Practice as the Negotiation of Dilemmas: An Analysis of the Conceptual, Pedagogical, Cultural, and Political Challenges Facing Teachers. https://doi.org/10.3102/00346543072002131, Review of Educational Research, 72(2), 131-175 (2002)

Wurdinger, S., Haar, J., y otros dos autores, A Qualitative Study Using Project-Based Learning in a Mainstream Middle School. https://doi.org/10.1177/1365480207078048, Improving Schools, 10(2),150-161 (2007)

Zancul, E. S., Sousa-Zomer, T. T., \& Cauchick-Miguel, P. A., Project-Based Learning Approach: Improvements of an Undergraduate Course in New Product Development. https://doi.org/10.1590/0103-6513.225216, Production, 27(spe), e20162252 (2017) 